\title{
Precise Orbit Determination and prediction of the ISS in the frame of the ACES Mission
}

\author{
Wermuth Martin, Montenbruck Oliver \\ German Space Operations Center (GSOC) \\ German Aerospace Center (DLR) \\ Oberpfaffenhofen, 82234 Weßling, Germany \\ martin.wermuth@dlr.de
}

\author{
Helm Achim \\ Astrium Space Transportation \\ 188039 Friedrichshafen, Germany \\ Cacciapuoti Luigi \\ European Space Agengy, Research and Scientific Support \\ Department (RSSD) \\ Noordwijk, The Netherlands
}

\begin{abstract}
The ESA (European Space Agency) fundamental physics mission ACES (atomic clock ensemble in space) on the ISS (International Space Station) has a high demand on orbit determination and orbit prediction accuracy to fulfill its scientific goals. Therefore the experiment will be equipped with a multifrequency JAVAD GNSS receiver. Due to constructional reasons, the GNSS antenna will be tilted from the zenith by $60^{\circ}$ at an azimuth of $50^{\circ}$. In addition the field of view of the antenna will be severely obstructed by the solar panels of the ISS. A simulation study using a GPS signal simulator and a JAVAD GNSS receiver demonstrates the impact of these limitations on a GPS orbit solution of the ISS. The observations recorded by the GPS receiver show small data gaps, but in general the data quality is good enough to generate a continuous orbit and reach the required accuracy.
\end{abstract}

Keywords: precise orbit determination (POD), ISS, GNSS, JAVAD receiver

\section{INTRODUCTION}

Atomic Clock Ensemble in Space (ACES, [1]) is a fundamental physics mission of the European Space Agency (ESA). It will consist of two atomic clocks - the cold-atom clock PHARAO and the space hydrogen maser SHM - which will be attached to the European Columbus module on the International Space Station (ISS). In order to fulfil the mission objectives, the orbit of the ISS has to be determined with an accuracy of better than $50 \mathrm{~m}$ in near real-time and better than $10 \mathrm{~m}$ in post-processing. Therefore the experiment is equipped with a JAVAD-Triumph GNSS receiver. During the last two decades, it has been shown for several low Earth orbiting (LEO) missions equipped with a GPS receiver like TOPEX/Poseidon, Jason 1, Jason 2 [3] [4], CHAMP [5] or GRACE [6], that precise orbit determination (POD) with an accuracy of $10 \mathrm{~cm}$ or better is possible. A recent study [2] using data from the Russian and American single-frequency receivers already on board the ISS, has shown that at least a $1 \mathrm{~m}$ accuracy can be reached with single-frequency GPS, and further improvements can be expected with dual-frequency receivers.

Nevertheless, the evaluation of GNSS observations from the ACES mission poses new challenges, as the field of view of the GNSS antenna is severely limited. Hence a GPS simulation using a realistic field of view mask was carried out to assess the accuracy which can be expected. The simulation was performed with a Spirent GSS7700 GPS signal simulator and a JAVAD Triumph GNSS receiver at the German Space Operations Center (GSOC).

This paper will provide a discussion of the accuracy requirements to the POD and describe the hardware of the GNSS subsystem and the architecture of the ACES Orbitography Center (ACES-OC).

\section{THE ACES ORBITOGRAPHY CENTER}

The transport of the ACES hardware to the ISS and its attachment to the Columbus module is currently planned for early 2016. The ACES-OC is implemented at the German Space Operations Center (GSOC), where also the Columbus Control Center (Col-CC) is located. The ACES-OC is responsible for producing three different orbit products based on the observations of the JAVAD GNSS receiver on a routine basis:

- a combined orbit fit and predicted orbit product, which is delivered in near real-time,

- a final high precision orbit product, which is delivered daily with a latency of one or two days, and

- a mid-term prediction product, which is delivered twice a day in the form of two-line-elements (TLEs). 


\section{A. Accuracy Requirements and Limitations}

The main objectives of the ACES mission are to test the predictions of Einstein's theory of relativity with unprecedented accuracy, to demonstrate the performance of a new generation of microwave clocks in space and to achieve time and frequency transfer with a stability of about $10^{-16}$. In order to perform relativistic clock corrections, the position and velocity vectors need to be known precisely. The once-perrevolution amplitude of the position error should not exceed $10 \mathrm{~m}$ in post-processing (e.g. with a latency of several days) and $50 \mathrm{~m}$ for quick-look purposes.

The time transfer between ground based atomic clocks and ACES will be carried out by microwave link (MWL) ground terminals and satellite laser ranging (SLR) stations. In order to plan contacts, the ground stations require daily mid-term orbit forecasts of the ISS of about $2 \mathrm{~km}$ accuracy. For this purpose, TLEs are provided. For actual pointing of the antennas and telescopes, the trajectory of the ISS needs to be known with an accuracy of $50 \mathrm{~m}$. The accuracy of the TLE model is not enough for this purpose. Hence short-term orbit predictions based on orbit determination results will be delivered in nearreal time at an update rate of $1.5 \mathrm{~h}$.

Although the POD concept using GPS observations is routinely operated on several low LEO missions, with accuracies much better than required for ACES, the POD for the ISS poses new challenges:

- Due to construction reasons the GNSS antenna will be attached to the ACES module with a tilt of $60^{\circ}$ from the zenith direction and of $50^{\circ}$ in azimuth direction. Hence the field of view is limited and asymmetric w.r.t. the flight direction, which results in a suboptimal geometrical distribution of the observed GPS satellites (see Fig. 1).

- The solar panels of the ISS further obstruct the field of view of the GNSS antenna limiting the number of observable GPS satellites to five in the worst case.

- The orbit height of the ISS is with approx. $350 \mathrm{~km}$ significantly lower than that of most LEO satellites. Combined with the large surface of the rotating solar panels, this makes modelling of air drag and thus the orbit prediction more difficult than for relatively compact satellites.

\section{B. Operational Orbit determination}

The ACES-OC will provide three types of orbit products. A combined orbit fit and predicted orbit product, a final orbit product and a medium term orbit prediction product.

The combined orbit fit and prediction product will be generated in near real-time and updated every $90 \mathrm{~min}$ and serves two purposes. The first one is to generate accurate position and velocity vectors for the computation of quick-look relativistic clock corrections. The second is to provide a short term prediction for SLR stations and MWL terminals. Hence the product will contain $6 \mathrm{~h}$ orbit fit to the observed data, and $6 \mathrm{~h}$ prediction.

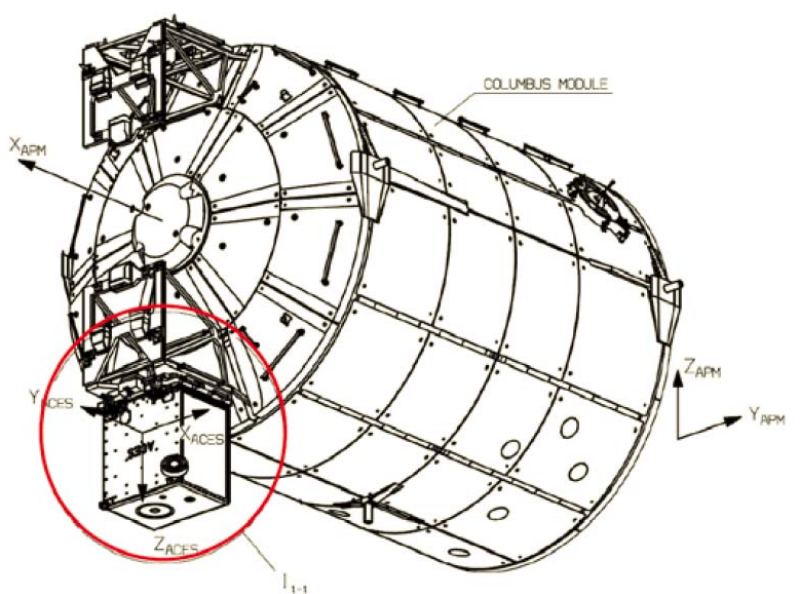

Figure 1. Position of the ACES module and the GNSS antenna.

In order to generate the near real-time products, the ACESOC receives all relevant observations from the ACES data stream via the Col-CC. The data will be provided in $5 \mathrm{~min}$ batches and assembled at the ACES-OC to cumulative products in order to reduce the transmission latency. The data package contains raw observations of the GPS, GLONASS and Galileo constellations recorded by the JAVAD GNSS receiver. As a backup for data gaps in the raw observations, the navigation solutions of the JAVAD GNSS receiver and the SIGI receiver [2] are provided. The ISS attitude information generated by the SIGI receiver is necessary for orbit determination as well.

The adjusted part of the combined product is computed using the most recent $6 \mathrm{~h}$ of raw observation data of the JAVAD receiver with a reduced dynamic orbit determination (RDOD). As a baseline, the IGS (International GNSS Service) ultra-rapid ephemeris product [7] will be used for the near-real time processing. As an alternative, use of DLR's RETICLE (Real-Time Clock Estimation [8]) product or the upcoming IGS real-time products is considered to further increase the quality of near real-time orbit determination. Subsequently a position fit (PosFit) is performed over the $6 \mathrm{~h}$ of precise orbit and propagated for $6 \mathrm{~h}$ into the future. The RDOD and PosFit software tools are part of the GHOST package (GPS High precision Orbit determination Software Tools [9]) developed at GSOC. Usually orbit determination products refer to the center of mass (CoM) of the spacecraft. In case of ACES, the orbit trajectory is transformed to the ACES reference point, which is located in the center of the microwave cavity of the PHARAO clock. This facilitates use of the resulting orbit product for the science processing and minimizes the impact of attitude uncertainties in the overall processing chain. The combined orbit fit and prediction product will be delivered in SP3 format [10] and in the consolidated prediction format (CPF [11]).

Twice a day, TLEs will be generated based on the results of the latest near real-time POD. These TLEs serve for mid-term planning of MWL and SLR station contacts.

With a latency of a few days, the final orbit product is computed. This period is used to fill potential downlink datagaps and to ensure the availability of high-quality GPS ephemerides. For the final orbit product, rapid GPS 
ephemerides [12] generated by the Center for Orbit Determination in Europe (CODE) will be used. Similar to the near real-time POD, the final POD is computed with the RDOD software - with the difference, that it is computed over predefined daily $30 \mathrm{~h}$ arcs with $3 \mathrm{~h}$ overlap to the previous and following days. The overlap periods enable an internal consistency check: if the difference between two overlapping arcs is too large, the automated processing is stopped and an operator has to generate the product manually.

\section{SiMULATION}

\section{A. Simulation Setup}

In order to asses the influence of the limited field of view on the visibility of GPS satellites and the expected orbit accuracy a simulation was set up. Artificial L1/L2 GPS signals for a receiver moving on a generic ISS-like trajectory were simulated by a Spirent GSS7700 signal simulator at GSOC (see Fig. 2). The simulator is controlled by a computer running the SimGEN simulation software. The generated signals were fed via a broadband LNA and a power divider to a JAVAD AlphaG3T receiver. The power divider caused a signal attenuation of $3 \mathrm{~dB}$. It was added for compatibility with another test that used two receivers. The observations of the receiver were recorded via an RS232-LAN converter on a laptop.

The simulated signals start at Jan. 12012 0:00 h. The simulation lasted for one week (GPS week 1669) and is based on the true GPS constellation of that week. GPS signals were generated for all satellites more than $5^{\circ}$ above the Earth tangent. In order to emulate the characteristics of the NovAtel 704x antenna [13], which is considered for the ACES mission, a $12 \mathrm{~dB}$ gain roll-off from zenith to the antenna plane was selected (see Fig. 3). The gain pattern is rotationally symmetric about the boresight axis and used for both the L1 and L2 frequency.

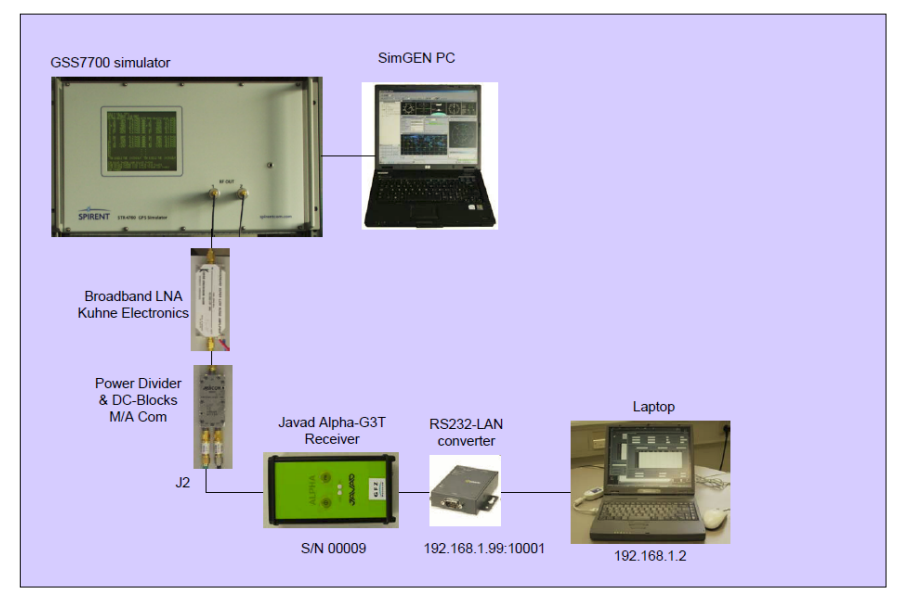

Figure 2. Simulation hardware setup.

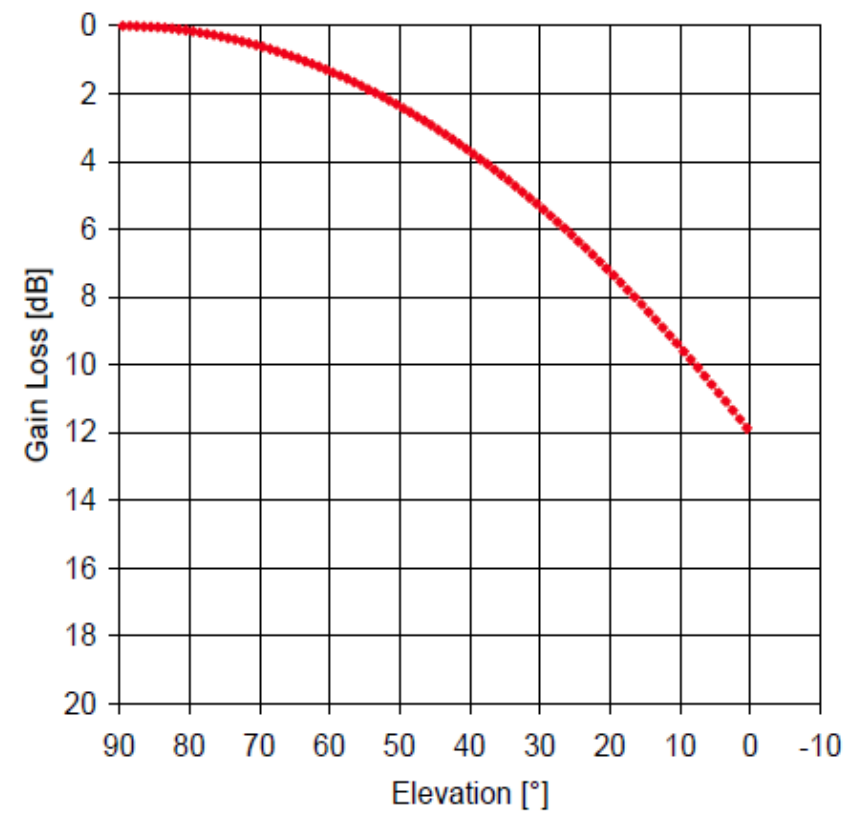

Figure 3. Elevaetion dependence of the antenna gain pattern.

A project internal study [14] has determined the obscuration of GNSS signals by the solar and heat radiation panels of the ISS. The degree of obscuration varies with the movement of the solar panels and has its maximum at a tilt angle of $135^{\circ}$. This worst-case has been used to create a fieldof-view mask (see Fig.4), which has been merged into the gain pattern. It should be noted, that the view in zenith-direction is blocked. Fig. 5 shows the antenna gain pattern in the local orbit frame: black marks the areas, which are obstructed by the ISS, red the region which is obstructed by the Earth. The brightest area is at the boresight (which is tilted from the zenith by $60^{\circ}$ at an azimuth of $50^{\circ}$ ) of the antenna indicating the highest gain.

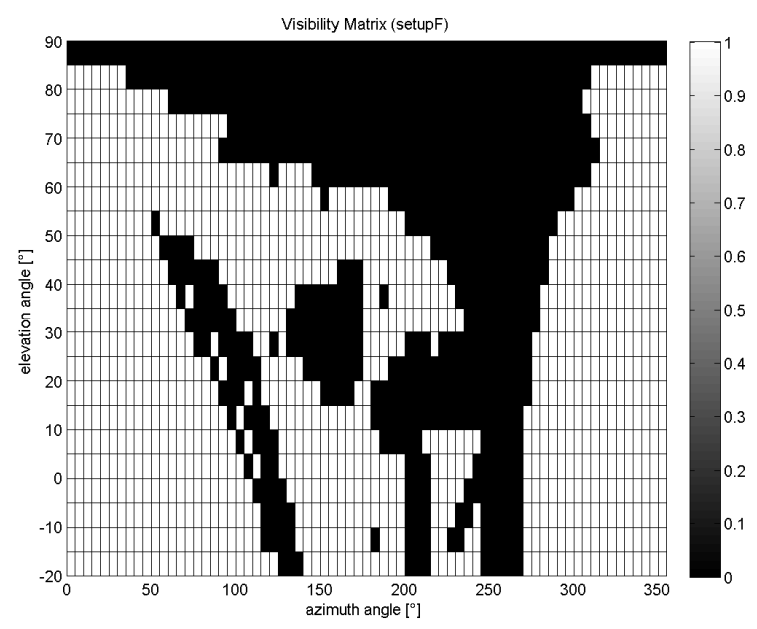

Figure 4. Field of view mask at $135^{\circ}$ solar panel tilt. 


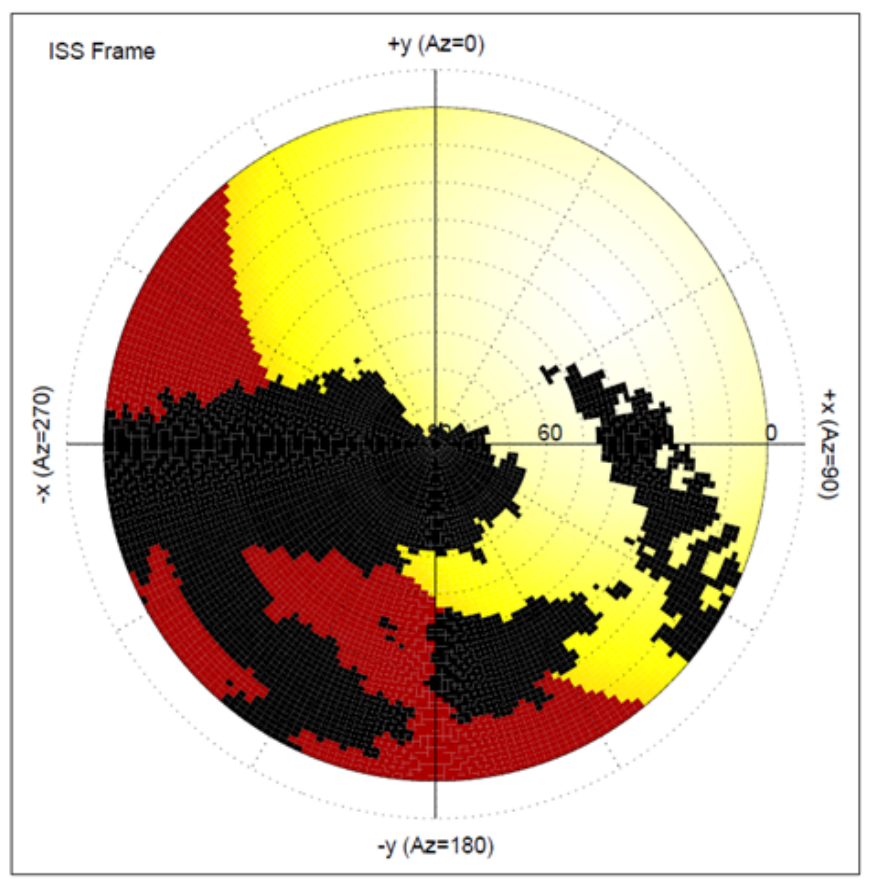

Figure 5. Antenna gain pattern in orbital frame.

The simulated ISS orbit was chosen to be similar to the actual ISS orbit. The orbit height was set to $350 \mathrm{~km}$, and the inclination to $51.6^{\circ}$. Only the right ascension of the ascending node was selected arbitrarily $\left(\Omega=100^{\circ}\right)$, so that the orbit trajectory starts near the zero meridian. The initial Keplerian elements for the simulation can be seen in Table 1. From then on, the orbit was determined by the SimGEN software by orbit propagation. Although, atmospheric drag plays a significant role for ISS orbit determination due to its large surface, atmospheric drag effects were disabled for the orbit propagation. This was done to avoid unwanted systematic effects, as that the simulation uses a different atmospheric modelling as the POD software.

TABLE I. SIMULATED ORBITAL ELEMENTS OF THE ISS

\begin{tabular}{|l|l|}
\hline \multicolumn{1}{|c|}{ Elements (J2000) } & \multicolumn{1}{c|}{ value } \\
\hline Epoch (GPS) & $\begin{array}{l}\text { 2012 Jan. 01, 00:00:00 } \\
\text { GPS week 1669, 0.0 s }\end{array}$ \\
\hline Semi-major axis $(a)$ & $6728.0 \mathrm{~km}$ \\
\hline Eccentricity $(e)$ & 0.001 \\
\hline Inclination $(i)$ & $51.6^{\circ}$ \\
\hline Right ascension of ascend. node $(\Omega)$ & $100.0^{\circ}$ \\
\hline Arg. of perigee $(\omega)$ & $45.0^{\circ}$ \\
\hline Mean anomaly $(M)$ & $315.0^{\circ}$ \\
\hline
\end{tabular}

The attitude of the ISS was simulated as strictly aligned with the orbital frame in a XVV orientation. This is a simplification, as the ISS sometimes actually adopts a pitch angle of about $5^{\circ}$, which further limits the field of view. In rare cases the ISS is even turned by $90^{\circ}$ to a YVV orientation. This assumption was necessary due to a lack of a proper attitude model of the ISS. Also vibrations on board the ISS were neglected, as no model was available. The ACES experiment, and thus the GNSS antenna will be placed several tens of meters from the ISS CoM. Hence vibrations could have a significant impact on the motion of the antenna and the orbit determination accuracy. The location of the ACES antenna was artificially chosen as $(+20 \mathrm{~m},+10 \mathrm{~m},+5 \mathrm{~m})$ in the spacecraft coordinate frame.

\section{B. Simulation Results}

A first comparison of the navigation solution produced by the JAVAD GNSS receiver with the simulated input orbit shows gaps and outliers of up to several hundred meters that are encountered in periods of bad GPS visibility and near initial acquisition of a navigation fix after outage periods. For about $1 \%$ of the epochs no navigation solution is available and for about $3 \%$ of the epochs the position error exceeds $10 \mathrm{~m}$. This shows that in these cases not enough satellites are visible to compute an accurate navigation solution. After removing the outliers (see Fig.6), the position errors show a bias of $0.07 \mathrm{~m}$ with a standard deviation of $1.02 \mathrm{~m}$ in the radial component, $0.04+/-0.66 \mathrm{~m}$ in along-track and $0.00+/-0.56 \mathrm{~m}$ in crosstrack. The 3D-RMS error of the navigation solution is $1.39 \mathrm{~m}$. The small bias of only $0.07 \mathrm{~m}$ of the radial component shows, that the dual-frequency navigation solution compensates very well for the effects of the ionospheric signal delay. With a simulated atmosphere of 20 TEC units, a radial bias of more than $10 \mathrm{~m}$ should be expected from a single-frequency navigation solution.

A look on the tracking statistics in Fig. 7 shows, that only during $0.3 \%$ of the epochs less than 4 satellites were tracked. Obviously the receiver cannot always provide a valid solution even when four satellites are tracked due to a temporary lack of broadcast ephemerides for newly acquired satellites. Fig. 7 also shows, that for more than $85 \%$ of the epochs six or more satellites are available, with an average of 7.1 satellites for both L1 and L2. This tracking behaviour is quite well considering that only satellites in the limited field of view, defined by the mask can be tracked (cf. Fig. 8).

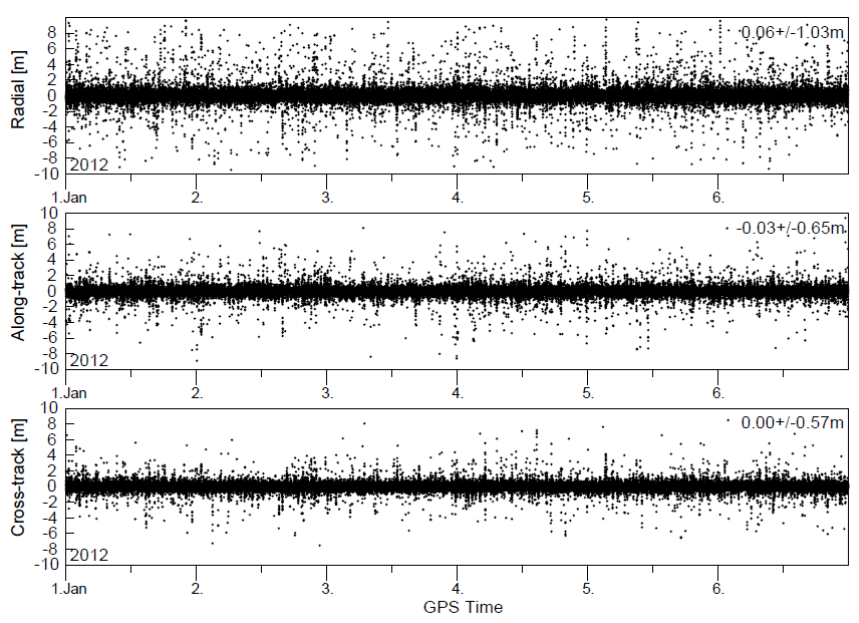

Figure 6. Errors of the dual-frequency navigation solution. 


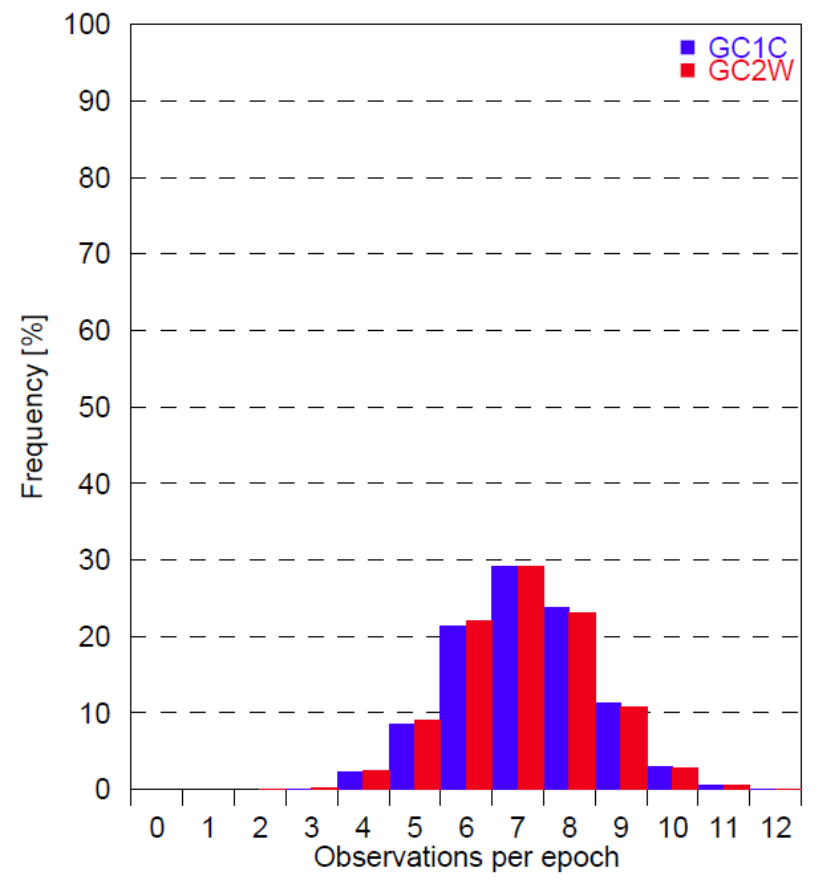

Figure 7. Tracking statistics for L1 and L2.

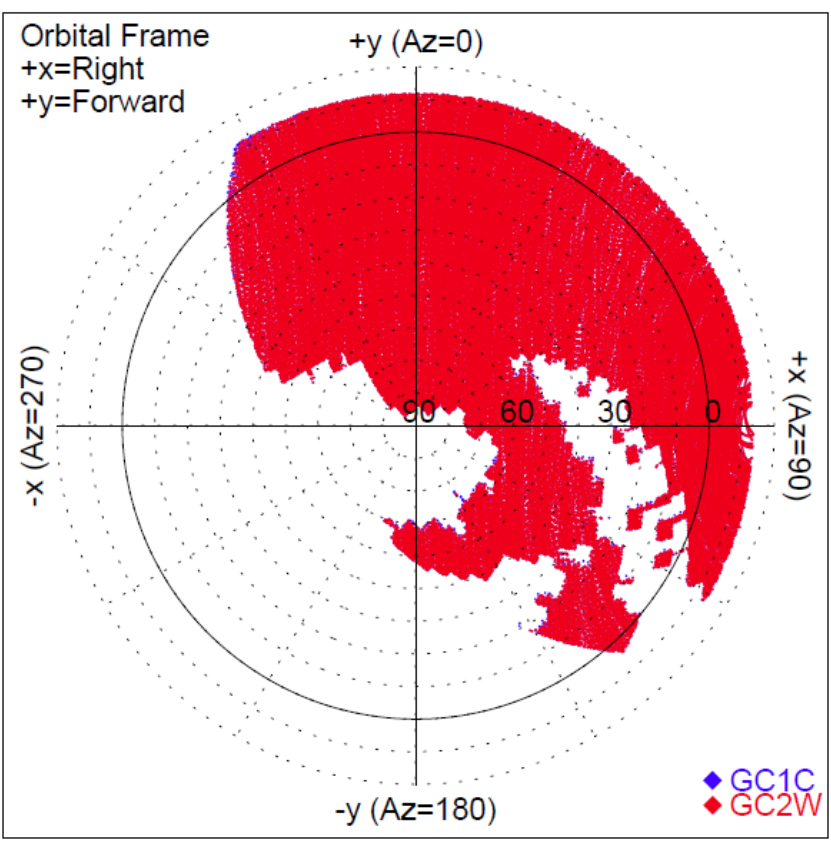

Figure 8. Location of observed satellites in anntenna plane.

The tracking sensitivity (Fig. 9) observed during the simulation by the JAVAD receiver reflects very well the characteristics of the antenna gain pattern (Fig. 3 and Fig. 5) used for the simulation. For the direct tracking of L1C and L2X code, the receiver achieves a peak carrier-to-noise ratio $\left(\mathrm{C} / \mathrm{N}_{0}\right)$ of $50 \mathrm{~dB}-\mathrm{Hz}$ and decreases gradually for lower elevations but is still larger than $35 \mathrm{~dB}-\mathrm{Hz}$ at an elevation of $0^{\circ}$. The observed carrier-to-noise ratios show a large dispersion. As the antenna boresight axis is not aligned with the zenith direction, geometric signal loss effects interfere with the antenna gain pattern. For the semi-codeless tracking of the encrypted Pcode, the $\mathrm{C} / \mathrm{N}_{0}$ vales $\mathrm{S} 1 \mathrm{~W}$ and $\mathrm{S} 2 \mathrm{~W}$ show an even stronger decrease towards the lower elevations. To evaluate the quality of the semi-codeless tracking quality, the size of the S1W-S1C and S2W-S1C difference is shown in Fig. 10. Both frequencies show an expected almost linear variation (in logarithmic scale) compared to S1C. The degradation of the signal due to semicodeless squaring losses increases with lower elevation.

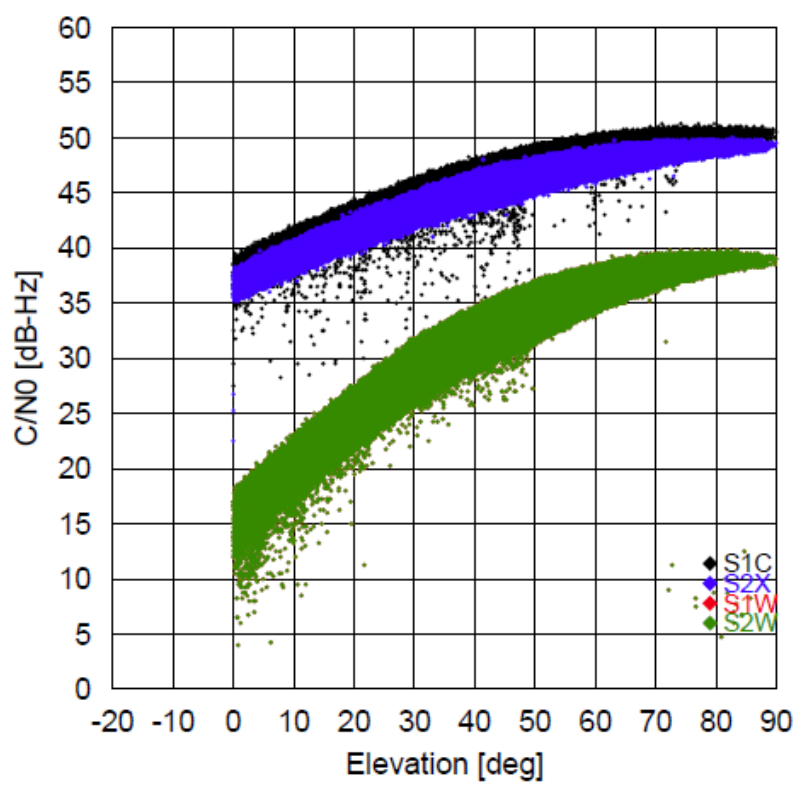

Figure 9. Tracking sensitivity.

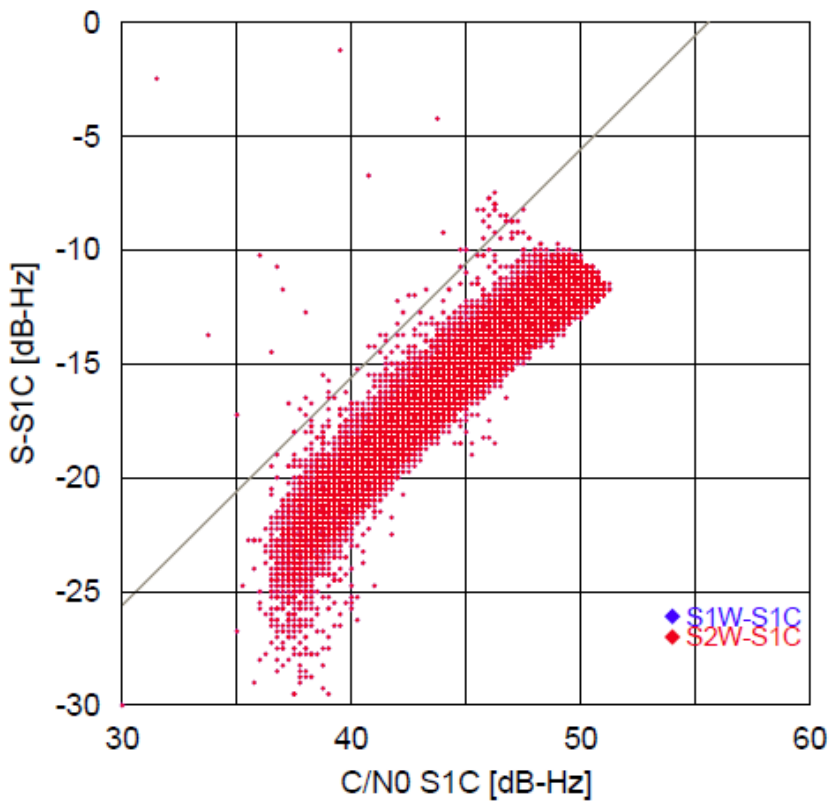

Figure 10. Semi-codeless tracking losses. 
The POD was computed in seven $30 \mathrm{~h}$ arcs with $6 \mathrm{~h}$ of overlap. The evaluation of the overlap periods shows a 3D position difference between two overlapping solutions of $1.5 \mathrm{~cm}$. Fig. 11 shows, that during the first and last half hours, there are edge-effects of several $\mathrm{cm}$, but apart from that, the overlapping arcs match to better than $1 \mathrm{~cm}$. This is not an exact measure of orbit accuracy, but an internal quality check, that hints to a high repeatability of POD results.

The a posteriori residuals (see Fig. 12) show an RMS of $0.77 \mathrm{~m}$ for code observations and of $6 \mathrm{~mm}$ for phase observations. The residuals for the carrier phase observations are largely driven by the noise of the semi-codeless $\mathrm{P}(\mathrm{Y})$ tracking. For comparison, phase residuals of only $2 \mathrm{~mm}$ would be obtained from direct tracking of the $\mathrm{L} 1 \mathrm{C} / \mathrm{A}$ and $\mathrm{L} 2 \mathrm{C}$ signals that could be considered in the simulation, but will not consistently be available on all satellites of the GPS constellation at the time of the ACES mission. The clock offset varies in a saw tooth pattern from $-150 \mathrm{~km}$ to $150 \mathrm{~km}$, which reflects the clock steering employed in the receiver. By default, the internal oscillator is allowed to drift freely and clock corrections in discrete $1 \mathrm{~ms}$ steps are performed once the offset exceeds an amplitude of $0.5 \mathrm{~ms}$. The employed test receiver exhibits a typical drift of almost $1 \mu \mathrm{s} / \mathrm{s}$ which results in a mean interval of $22 \mathrm{~min}$ between consecutive clock offset adjustments. Within the ACES project, the receiver can be driven by the highly stable ACES clock signal instead of the internal oscillator. This will effectively remove the need for clock offset corrections.

Finally, the resulting orbits are compared to the simulated input orbit (see Fig. 13). The differences are a direct measure of the POD accuracy that can be achieved under ideal conditions. The errors amount to $0.01+/-0.01 \mathrm{~m}$ in the radial component, $0.00+/-0.01 \mathrm{~m}$ along-track and $0.02+/-0.02 \mathrm{~m}$ cross-track or $0.03 \mathrm{~m}$ in 3D-RMS. This is at the level of accuracy which is usually achieved for LEO missions and well below the required accuracy of $10 \mathrm{~m}$. On the other hand, the simulation does not account for error sources such as multipath, attitude uncertainties or phase pattern distortions. Some degradation of the true flight performance with respect to the simulation results will therefore have to be expected.

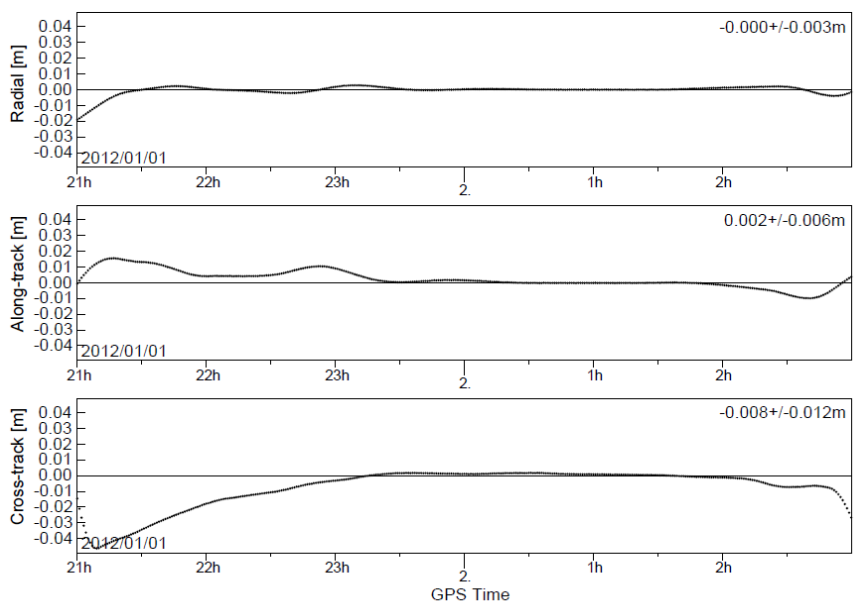

Figure 11. Orbit differences between overlapping POD arcs.

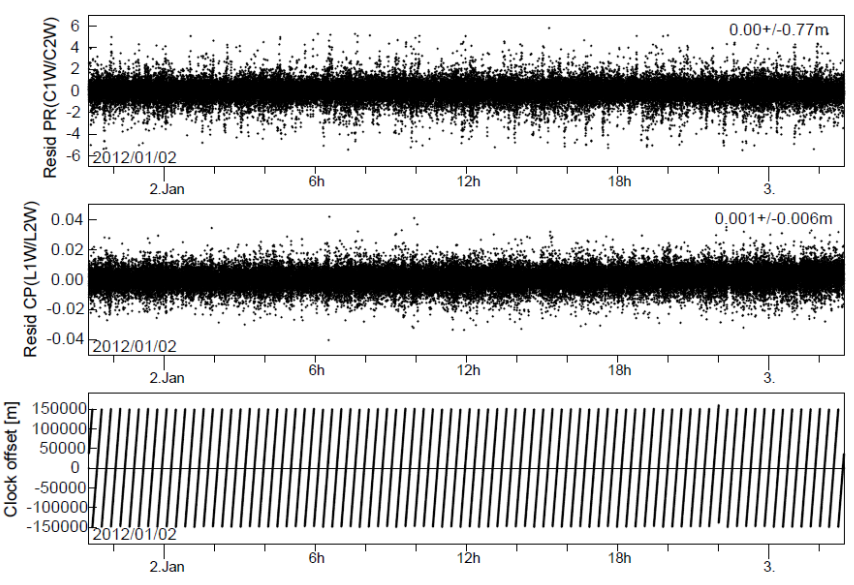

Figure 12. GPS residuals.

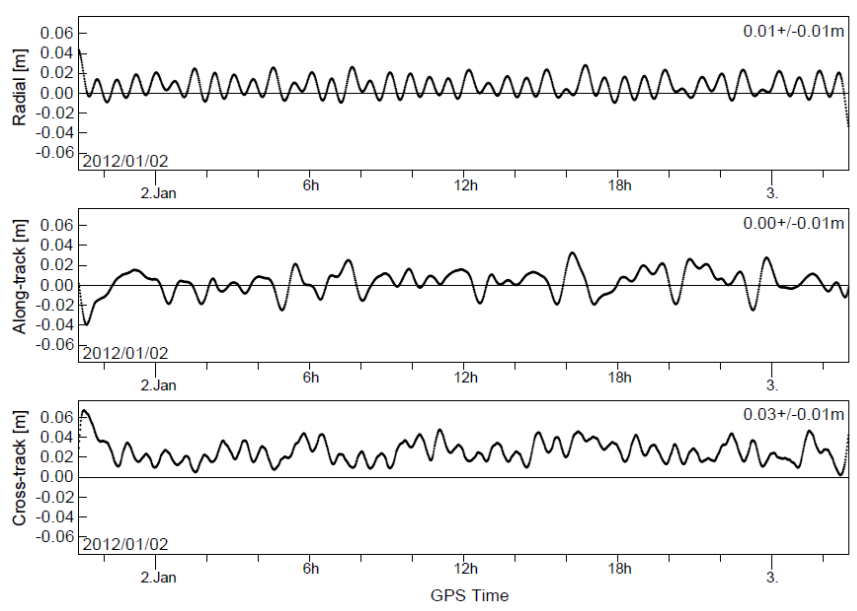

Figure 13. Orbit determination errors.

\section{CONCLUSIONS AND OUTLOOK}

The simulation results have shown that the JAVAD Delta G3TH receiver selected for the ACES mission is well capable to acquire and track GPS satellites under the specific conditions of high orbit dynamics and frequently interrupted signals. The receiver could not provide an accurate navigation solution in only $4 \%$ of the epochs. None of the gaps are long enough to seriously disturb the POD. The orbit comparison suggests that an orbit accuracy at the decimeter level or better will be achievable in the absence of severe multipath or dynamic perturbations of the ISS.

On the one hand, several effects including atmospheric drag, attitude variations of the ISS, vibrations of the ISS structure and multipath effects have been neglected due to the lack of proper models. These effects surely disturb a POD solution. In order to improve the simulation, assumptions on these effects could be derived from an analysis of the data from the receivers, which are already on board.

On the other hand, tracking of the GLONASS constellation and of available Galileo satellites, should significantly increase 
the number of available satellites, and enable the receiver to deliver uninterrupted data. Hence a further simulation study should incorporate GLONASS and/or Galileo.

\section{REFERENCES}

[1] L. Cacciapuoti and C. Salomon, "Space clocks and fundamental tests: the ACES experiment," The European Physical Journal Special Topics 172: 57-68 (2009). doi: 10.1140/epjst/e2009-01041-7.

[2] O. Montenbruck., S. Rozkov., A. Semenov., S. F. Gomez.., R. Nasca and L. Cacciapuoti, "Orbit Determination and Prediction of the International Space Station," Journal of Spacecraft and Rockets, 48, 6, 1055-1067, doi: 10.2514/1.52657, 2011.

[3] L. Cerri, J. P. Berthias, W. I. Bertiger, B. J. Haines, F. G. Lemoine, F. Mercier, J. C. Ries, P. Willis, N. P. Zelensky and M. Ziebart, "Precision orbit determination standards for the Jason series of altimeter missions," Marine Geod., 33(S1), 379-418, 2010.

[4] F. G. Lemoine, N. P. Zelensky, D. Chinn, D. E. Pavlis, D. D. Rowlands, B. D. Beckley, S. B. Luthcke, P. Willis, M. Ziebart, A. Sibthorpe, J. P. Boy, and V. Luceri., "Towards development of a consistent orbit determination, TOPEX/Poseidon, Jason-1, Jason-2," Adv. Space Res., 46(12), 1513-1540, 2010.

[5] C. Reigber, H. Luhr and P. Schwintzer, „CHAMP mission status.“ Adv Space Res 30 (2): 129-134, 2002.

[6] B. D. Tapley, S. Bettadpur, J. C. Ries, P. F. Thompson and M. M. Watkins, "GRACE measurements of mass variability in the Earth system.” SCIENCE 305 (5683): 503-505, 2004.
[7] J. Dow, R. E. Neilan and C. Rizos, "The International GNSS Service in a changing landscape of Global Navigation Satellite Systems," J. Geod., 83(3-4), 191-198, 2009.

[8] A. Hauschild, O. Montenbruck, "Real-time Clock Estimation for Precise Orbit Determination of LEO-Satellites," Proceedings IONGNSS-2008 Conference, 581-589, A2b4, 16-19 Sept. 2008; Savannah, Georgia, 2008.

[9] O. Montenbruck, T. van Helleputte, R. Kroes and E.Gill, "Reduced Dynamic Orbit Determination using GPS Code and Carrier Measurements," Aerosp. Sci. Technol. 9/3, 261-271, DOI 10.1016/j.ast.2005.01.003, 2005.

[10] S. Hilla, "The Extended Standard Product 3 Orbit Format (SP3-c)," NOAA (2007), http://www.ngs.noaa.gov/orbits/sp3c.txt

[11] R. L. Ricklefs, "Consolidated Laser Ranging Prediction Format, Version 1.01," The University of Texas at Austin, Center for Space Research, 2006.

[12] R. Dach, E. Brockmann, S. Schaer, G. Beutler, M. Meindl, L. Prange, H. Bock, A. Jäggi and L. Ostini, "GNSS processing at CODE: status report," J. Geod. 83 (3-4), 353-365, doi:10.1007/s00190-008-0281-2, 2009.

[13] NovAtel Inc., "GPS-704X Antenna Design and Performance," http://support.novatel.com/entries/315180-gps-704x-antenna-designand-performance; last accessed: 13 July 2012.

[14] A. Helm A, "ACES GNSS Signal Reception Performance," ACE-TN13300-007-AST; Issue 1.0, 31 May 2011. 